\title{
Erotica mariniana
}

\section{Francesco Guardiani}

Fino a pochi anni fa parlando di opere secentesche ci si poteva riferire ad esse come a luoghi ignoti, o addirittura malfamati, della nostra letteratura. Oggi la situazione è cambiata, ma il lavoro di risanamento (avviato in modo sistematico da Giovanni Pozzi nel 1960$)^{1}$ può dirsi tutt'altro che completo. Il recupero dei testi, premessa indispensabile per ogni possibile mutamento di orientamento critico sul secolo, si va effettuando soprattutto nell'attrezzatissima officina svizzera di Friburgo e, in Italia, presso vari nuclei universitari recentemente coalizzati nell'impresa "Archivio Barocco." In questo fervido lavoro di riscoperta si privilegia giustamente l'opera del "re del secolo."2 Abbiamo già L'Adone, Le dicerie sacre, La strage degl'Innocenti, La galeria, la Gierusalemme distrutta; ${ }^{3}$ è quasi pronta per la stampa l'attesissima Lira di Alessandro Martini e Ottavio Besomi, e non mancano i curatori alla Sampogna, agli Epitalami, al Ritratto, alle "rime burlesche" e alle altre opere minori.

Non c'è stato ancora, che io sappia, chi abbia intrapreso l'esplorazione della selva infida dei componimenti erotici che stanno al di fuori delle opere date a stampa dal poeta stesso. Selva infida perché nello scorrere queste poesie lo spettro dell'apocrifo è sempre in agguato. La leggenda del Cavalier Marino, si sa, è legata a non poche pubblicazioni in busta chiusa. ${ }^{4} \mathrm{E}$ in busta chiusa, appunto, non si va per il sottile: perfino componimenti stilisticamente sospetti, o addirittura scorretti, sono stati attribuiti senza la minima perplessità al Marino. Probabilmente quando la nebbia si sarà diradata, quando cioè, restituiti ai testi originari i brani dell'Adone, della Lira e della Sampogna, dalle raccolte di "rime erotiche" saranno stati espunti gli apocrifi, si resterà con un pugno di mosche e non sarà il caso di pensare ad un'edizione critica. Ma intanto il lavoro di spoglio e di accertamento va fatto. Esso dovrà procedere tenendo conto da una parte dei vari aspetti della lingua e dello stile, dall'altra del pensiero del poeta in fatto 
di sessualità. ${ }^{5}$ È di questo secondo corno del problema che mi occupo nelle pagine che seguono. Rimando ad altra sede la serie inivitabile di confronti tra ciò che è del Marino e ciò che potrebbe esserlo e procedo ora tentando di precisare la logica del poeta nella sua concezione dell'amore e, da qui, di far luce sulla ragione del suo erotismo.

Da piú parti è stata rilevata nella poetica del Marino l'assenza di una chiara linea di sviluppo: dalla Lira I (1602) all'Adone (1623) i soli punti precisi di riferimento per cogliere modifiche, trasformazioni, nuovi interessi insomma, nello stile e nella scelta dei temi, sembrano essere le date di pubblicazione delle numerose opere apparse nel glorioso ventennio. Date che poi non dicono neanche molto perché, è accertato, il poeta lavorava contemporaneamente a piú opere, rimaneggiava continuamente i suoi componimenti e li teneva nel cassetto per anni prima di darli alle stampe. Un nuovo vaglio dell'Epistolario ${ }^{6}{ }^{6}$ notoriamente avaro in fatto di dichiarazioni esplicite di poetica, contribuirà senz'altro, nelle mani dell'agguerritissimo Giorgio Fulco, a chiarire piú di un punto oscuro. Visto comunque lo stato attuale delle ricerche sulla biografia poetica del Nostro, decido di trattare la sua intera produzione come un tutt'uno dando, però, particolare rilievo all'Adone, ultima e completa definizione di un mondo poetico variamente tratteggiato nelle opere precedenti.

L'erotismo del Marino, cioè la tendenza a trattare, rappresentare e perfino esaltare l'amore sessuale, nasce dallo sblocco di una situazione poetica, di antica tradizione, in cui la donna è contemplata e cantata come un oggetto irraggiungibile del desiderio. In tale tradizione la passione amorosa coincide con una perenne ricerca di valori assoluti (da cui l'identificazione di ideali laici con ideali religiosi) programmaticamente dissociati da ogni "conquista," o "possesso," materiale. Con Marino cade definitivamente e, direi, ufficialmente la concezione della donna come figura ideale di guida o di riferimento nella ricerca di un'eterea perfezione. La donna è raggiungibile e spesso raggiunta per cui il rapporto sessuale diventa una realtà dell'esercizio amoroso e quindi una situazione "cantabile." Di quanto questa nuova percezione abbia demitizzato la figura della donna può rilevarsi bene in questo verso:

Per la PORTA del Ciel vassi all'Inferno. ${ }^{7}$ 
La "porta del Ciel" è un'immagine neoplatonica trasposta dal suo senso originario al significato di sesso femminile. La tendenza alla conquista della donna non porta piú al Paradiso tout court, ma ad un ambiguo

Paradiso infernal, celeste Inferno. ${ }^{8}$

Noto, di passaggio, un'ombra di chiesistica moralità in questi ossimori. Mi pare, comunque, e risulterà piú chiaro dalle pagine seguenti, che il poeta voglia qui indicare qualcosa di piú: lo stato angoscioso della condizione umana in equilibrio instabile tra due realtà, quella del mondo sensibile e quella del mondo ideale.

Molti lettori del Marino, anche in tempi recenti, si sono fermati all'osservazione della caduta del velo della castità ed a ciò che questo comporta nelle situazioni amorose presentate dal poeta. Con questa prospettiva ridotta si è dato massimo rilievo ad elementi quali: la dinamica della seduzione (rilevabile, per esempio nella canzone "Trastulli estivi" ${ }^{\prime \prime}$ che è, non a caso, tra i componimenti piú citati); l'allargamento del codice descrittivo riferito all'anatomia della dónna (oltre ai consueti "occhi," "capelli," "labbra," "viso," Marino tratta anche di "gambe" e di "seno"); la gestualità dell'amplesso (basti ricordare l'attività delle due coppie ninfa-satiro, Venere-Adone nel canto VIII del poema). Si è giunti cosí ad associare all'erotismo mariniano un massiccio impoverimento spirituale; detto altrimenti, a ridurre l'intera proiezione di un mondo ideale prospettato dal poeta al "miserrimo" appagamento sessuale. Se l'ideale è raggiungibile e raggiunto, ha ragionato Getto, la poesia del Marino

appare, fondamentalmente, sgorgata da un'anima serena, da un'esperienza di vita prosperosa e soddisfatta, ponendosi come il prezioso ornamento di una esistenza tranquilla, come una splendida superfluità di una vita molle e lussuosa. L'espressione lirica non è mai, come per altri poeti (forse per i soli grandi poeti) una necessità interiore, un rifugio tra i mali dell'esistenza, una missione e un messaggio di vita. ${ }^{10}$

(Il finissimo interprete del Tasso è rimasto sordo al richiamo del cigno di Sebeto). Questa stessa percezione di base, pur se riscontrata da una angolazione del tutto diversa, è stata condivisa piú recentemente dall'Asor Rosa. ${ }^{11} \mathrm{Ma}$ nei tempi nuovi della critica mariniana un'interpretazione del genere appare ormai insostenibile. Si è scavato a fondo nella psicologia poetica del Marino e 
ne è risultata una figura complessa e sfumata, impossibile da liquidare con le tinte forti, o meglio con i bianchi e i neri, di stampo ancora crociano. ${ }^{12}$

Ciò che osta ad un'interpretazione riduttiva e, tutto sommato, semplicistica dell'erotismo marianiano è una problematica insoddisfazione che il poeta denuncia immancabilmente ogni volta che dà una rappresentazione del rapporto sessuale. L'insoddisfazione non è causata dalla mancanza di appagamento fisico (anche se questo può avvenire, come nel caso degli "Amori notturni" o del "Duello amoroso"), ${ }^{13}$ ma dalla coscienza dell'impossibilità di un totale appagamento del bisogno d'amore che è anche un bisogno spirituale. L'atto sessuale è una necessità nella poetica del Marino che mira ad una comunione perfetta, materiale e spirituale, nell'amore; ecco perché, anche quando esso può considerarsi fisicamente riuscito (solo fisicamente), non dà pace al poeta, non lo libera dall'ansia smaniosa per la conquista di ciò che è veramente completo e definitivo. Nella già citata canzone "Trastulli estivi," alla fine dell'elaborato rituale della seduzione, egli ricorre allo sberleffo per sfogare la sua insoddisfazione. Ecco il famoso commiato:

\section{Canzon, lasciar intatta}

Da sé partire amata Donna e bella

Non cortesia, ma villania s'appella.

Se con l'amplesso egli veramente avesse avuto la sensazione di raggiungere il suo ideale, avrebbe fatto suonare le campane a distesa invece di erompere in una dichiarazione cosí cinica. La quale, intanto, ci dà un'indicazione preziosa del fondamentale risentimento del poeta nei confronti della donna che qui, come altrove, non è un'amante identificata o identificabile, ma è la donna in generale. È sua la "colpa" se l'amore totale rimane solo un desiderio e non si attualizza mai. Vediamo perché.

Nell'esercizio amoroso, per Marino, concorrono due fattori essenziali, l'istinto e la ragione. L'istinto corrisponde a quella carica viscerale comune a tutti gli elementi del creato; è l'unica forza propellente dell'amore degli animali, delle piante e perfino delle pietre come risulta dallo splendido inno alla natura della musa Talia che conclude il VII canto dell'Adone. Nell' amore umano l'istinto è associato alla condizione della gioventú: tutti gli amanti del Marino sono giovani, il poema grande è dedicato a "giovani amanti e donne innamorate, "14 i vecchi (ma soprattutto le vecchie) 
sono derisi e biasimati finanche con l'invettiva (si pensi a Sofrosina, a Feronia, alla Grifa e al vecchio sdentato protagonista di un madrigale variato sul tema del "bacio mordace"). ${ }^{15} \mathrm{Ma}$ l'elemento peculiare che distingue l'amore umano da quello degli esseri inferiori è la ragione. È proprio qui, nel territorio della ragione, che si apre la spaccatura insanabile tra l'uomo e la donna, il maschio e la femmina.

La ragione, per Marino, non dipende dal desiderio di raggiungere una comprensione scientifica della natura, per quanto questo analito rientri nella quest intellettuale del poeta, ma è direttamente collegata e profondamente radicata al senso di meraviglia percepito di fronte al "miracolo della vita." Come ha meglio di ogni altro spiegato Ardengo Soffici, un lettore occasionale ma ispirato del Marino, la vera meraviglia del poeta corrisponde alla capacità di "prolungare in sé lo stato infantile, vergine, dei sensi e dell'anima" che lo pone "in grado di percepire le sfumature infinite, le varietà impercettibili, il divino continuo inedito della misteriosa rappresentazione" della vita. ${ }^{16} \mathrm{Si}$ nota chiaramente come questo commosso stupore si sviluppi nella pagina mariniana con il sostegno della ragione in numerosi componimenti. Ricordo quelli intestati alla "Lontananza," al "Sogno," al "Pensiero": non sono mai puri slanci emotivi o astratte operazioni della mente, ma vere e proprie intimizzazioni cerebralizzate del sentimento. Il "Pensiero" del Marino "dietro al vago desio che gli fa scorta/Dal fondo del cor ... si leva a volo."17 La donna ha in sé lo stesso "vago desio," ma è incapace di volare. Il perché di questa limitazione è spiegato chiaramente in questi versi dell'ultimo "idillio favoloso" della Sampogna, "Piramo e Tisbe";

... perché 'n quel sesso

Minor naturalmente

Suol esser la ragione. ${ }^{18}$

C'è quindi nella natura umana stessa un fallo, un errore, che Marino rileva quasi leopardianamente considerando il suo tempo, non a caso definito spesso "secol rio," "empio," "guasto." A renderlo tale è stata la caduta della speranza che veniva dalla fede nell'ideale platonico. Il Pieri, in uno dei suoi consueti lampi di penetrazione critica, ha segnalato quest'estremo limite della poesia mariniana registrando, accanto allo "scontento per la pomposa falsità delle teorie dell'amore platonico," un angoscioso senso di "inadeguatezza alla vita."19 
Ma da qui, da questa desolata visione della condizione umana, Marino non si lascia andare nel baratro del pessimismo, né sceglie à la Boccaccio (del Corbaccio) la caustica via della misoginìa se pure, va notato, spesso riserva alle donne gli epiteti più acri: crudele, insensibile, venale, sciocca, vanitosa, rozza et cetera. La soluzione di Marino, e se non m'inganno è questo che lo rende un poeta "attuale," particolarmente vicino, cioè, alla nostra sensibilità post-moderna, consiste nell'accettazione dei limiti della natura umana sfuggendo l'ormai scoperta fallacia delle riduzioni ad un assoluto nella cui contemplazione egli avverte che il senso è inerte e l'intelletto si perde. ${ }^{20} \mathrm{Di}$ nuovo, con questo, si ripresenta lo spettro di un materialismo superficiale. Ma per Marino, entro i limiti della natura umana, così come c'è un corpo fatto di carne ed ossa, c'è anche un bisogno inalienabile di valori assoluti. Per questo egli si muove, coerentemente, a ricercarli con gli occhi sbendati ed i piedi per terra. Il suo tentativo di "congiungere l'incongiungibile, di conciliare l'inconciliabile," come ha notato il Calcaterra, 21 illustra proprio la sua quest dell'ideale poggiata sul fermo rifiuto di prescindere dal peso fisico dell'umanità.

Tasso aveva tentato nel Mondo creato di risolvere una dicotomia simile a questa fidando in una soluzione palingenetica - evidente nel rilievo assegnato alla figura della fenice - che comunque rimandava la purificazione ad un tempo futuro (magari prossimo, ma pur sempre futuro), alla fine del mondo. Marino è un poeta dell'oggi, invece: sentendosi negato l'assoluto, cerca appagamento in quelle briciole d'assoluto che sono le epifanie brevissime e fuggevoli: i "piaceri imperfetti," i "pensieri," i "sogni," gli amplessi e i "baci." L'ars amandi di Marino, in fondo, non è che il tentativo di dilatare, razionalizzandole, le effusioni spontanee e immediate del sentimento. Questo si nota bene, per esempio, nel seguente dialogo tra Adone e Venere:

- Dimmi o dea (dice l'un) questi tuoi b
movon così dal cor, come dal labro? -
Risponde l'altra: - Il cor nele mordaci
labra si bacia, amor del bacio è fabro,
il cor lo stilla, il labro poi lo scocca,
il più ne gode l'alma, il men la bocca. ${ }^{22}$

$\grave{E}$ proprio in queste elegantissime cerebralizzazioni, tralasciando per un attimo l'amore di Venere e Adone meritevole di un discorso a parte, che si ha il segno di un aspetto importantissimo dell'erotismo mariniano: l'amore "a solo," condotto cioè soltanto 
da parte e dalla parte dell'uomo. Così, per esempio, nell'idillio di Atteone, ${ }^{23}$ così nel caso del notturno visitatore del tempio di Venere che eiacula sulla statua della dea lasciando "la macchia in sul bel fianco impressa." 24 Non è un caso, dunque, che la vista, come stimolo dell'intelletto, giochi un ruolo maggiore nell'esercizio dei sensi. Marino, sappiamo dall'epistolario, "impazzisce" per la pittura e "vagheggia" a lungo, rapito, la Venere nuda del Castello. ${ }^{25}$

Più che all'appagamento sessuale egli mira alla conquista "ragionevole" del quid che genera il sentimento amoroso. Anche l'omosessualità è spiegata dalla ragione. Nella canzone "Contro il vizio nefando," 26 che forse voleva essere una risposta all'accusa dei suoi detrattori che glielo rimproveravano, in fondo egli finisce per giustificare l'amore infecondo riconoscendolo come un prodotto del secolo, il secolo "rio," tale appunto perché ha segnato un deciso prevalere della ragione sull'"irragionevole," ma confortante, platonismo. Così, razionalizzando e demistificando, Marino accetta praticamente ogni forma d'amore purché in esse si scorga il luccichío di un'umanità "intellettualmente" sensibile e disponibile a giocare il suo ruolo nel "gran theatro del mondo."

Ma la più chiara, coerente e completa illustrazione mariniana di poetica amorosa è nell'Adone. Il poema si può leggere come un doppio teorema: dalla condizione umana deriva l'impossibilità di conquistare l'assoluto; da questa situazione angosciosa deriva la possibilità di risalire la china e raggiungere uno stato di pacato ottimismo senza illusioni. Una prima considerazione di base per seguire questa interpretazione riguarda il riconoscimento nell'opera di due parti ben distinte, separate dalla discesa, o meglio "caduta," di Adone dal Cielo di Venere all'Inferno di Falsirena. ${ }^{27}$ La prima parte è costruita sul modello platonico dell'elevazione dell'uomo al cielo: così Adone, povero naufrago alla deriva, seguendo la Bellezza è innalzato per gradi fino al paradiso. La seconda parte corrisponde alla demolizione sistematica dell'impianto platonico. Nella prima c'è il mondo come l'uomo vorrebbe che fosse, nella seconda c'è il mondo così com'è. Troviamo qui un continuo alternarsi di alti e bassi, di fortuna e sfortuna, di sollievo e angoscia, il tutto ridotto nell'ambito della più piatta umanità. Qui l'amore di Venere e Adone, cantato solennemente nei "Trastulli," viene a mostrare anch'esso i suoi limiti "umani." Ecco come. Avvertito da mille presagi svagatamente trascurati, Adone improvvisamente si scuote di dosso il consueto torpore. Il suo personaggio nel canto XVII prende tinte decisamente eroi- 
che. Caduta ogni illusione di poter perpetuare lo stato edenico, così si rivolge alla dea che si accinge a partire e, praticamente, a lasciarlo morire:

Veggio or ben io che dal tuo figlio avaro qualche breve talor gioia s'ottiene sol perché cresca alfin lo strazio amaro e si raddoppi il mal, perdendo il bene.

La risposta di Venere chiarisce precisamente la ragione dell'angoscia mariniana:

Ma che poss'io se mi rapisce e move violenza fatal di legge eterna? (XVII, 26)

Eccolo, dunque, nei suoi effetti, quel fallo della natura umana di cui dicevo sopra: su ogni vita, su ogni amore, perfino su un amore immaginato poeticamente come perfetto e ideale, incombe l'ombra sinistra del fato, una forza misteriosa e incontrollabile che non si può identificare né con il Giove del poema, vecchio libertino che ruba i baci a Ganimede, ${ }^{28}$ né con il Dio cristiano dell'apocalissi dei Gesuiti, come ha chiarito eloquentemente il Calcaterra. ${ }^{29}$

Ma Marino, s'è detto, non è un poeta dell'angoscia. Egli va oltre l'angoscia. Il fato conduce alla morte, ma dopo la morte ritorna la vita. La vicenda di Adone si conclude in una nuova primavera nella quale il giovinetto ucciso rinasce come fiore. L'evidenza della metamorfosi diventa la prova di una umana immortalità. La pacata serenità che da questa certezza deriva porta il poeta ad una nuova straordinaria consapevolezza del valore dell'effimero: ogni drappo colorato, ogni armatura luccicante, ogni cosa che si muove nel caleidoscopico ultimo canto è percepita come parte attiva di un mondo che vive di se stesso, che si compiace della luce che riesce a produrre da solo, senza illusorie illuminazioni divine.

Estremamente significativo dell'ultimo approdo poetico-speculativo del Marino - e singolare il fatto che fino ad oggi non se ne sia parlato - è lo strano discorso accademico pronunciato dal poeta di fronte agli Oziosi di Napoli poco prima della morte. ${ }^{30}$ L'esplicito accenno che lì si fa a Pitagora, alla teoria della trasmigrazione delle anime, mentre suggerisce all'erudito insospettabili (ma non troppo: Galeria) incontri con il conterraneo Della Porta, 
anche al più distratto lettore del poema non può che mostrare ora la conferma di una concezione dell'universo come organismo gravido di vite interrelate, che si incontrano e si compensano nel piacere e nel dolore, nel loro continuo movimento prodotto dalla forza misteriosa dell'Eros. Un movimento che dunque non è un progressivo divenire con uno scopo finale, ma un gioco senza vinti né vincitori, in cui tutti sono premiati, come negli "Spettacoli" dell'ultimo canto, per mano della dea dell'Amore, l'unica vera divinità che può donare in terra

di pacifico stato ozio sereno. ${ }^{31}$

University of Toronto

NOTE

1 Giovan Battista Marino, Dicerie sacre e Strage degl'Innocenti, a cura di Giovanni Pozzi (Torino: Einaudi, 1960).

2 La definizione è del De Sanctis. In Storia della letteratura italiana (Milano: Feltrinelli, 1970), cap. XVIII, P. 639.

3 Dell'Adone abbiamo due ottime moderne edizioni, quella curata da Giovanni Pozzi (Milano: Mondadori, 1976) e quella curata da Marzio Pieri (Bari: Laterza, 1975-1977). Delle Dicerie e della Strage s'è detto sopra. La galeria è stata riproposta dal Pieri (Padova: Liviana, 1979) e cosí la Distrutta (Parma: La Pilotta, 1985), primo volume dell'"Archivio Barocco."

4 Il corpus dei componimenti erotici attribuiti a Marino non è stato ancora ricostituito. Elenco qui, in ordine cronologico le schede bibliografiche delle opere finora raccolte: "Poesie erotiche," in Opere del Cavalier G.B. Marino (Napoli: C. Boutteaux e M. Aubry Editori, 1861); La primière nuit de marriage (Parigi?: Au Palais-Royal, 1883); Venere pronuba, Gli amori notturni, $i$ Baci ed altre poesie (Milano: ?, 1883); Il nuovo giardino d'amore (Napoli: ?, 1886); Poesie erotiche. Libro chiuso per adulti del Cav. Marino (Napoli: Rideri, 1888); Scuola di Voluttà, ovvero segreti e misteri dell'amore (Napoli: ?, 1890); Poesie classiche erotiche (Roma: Capaccini, 1896); Angelo Borzelli, Il Cav. G.B. Marino (Napoli: Priore, 1898), contiene il componimento "Amoroso Trattenimento del Cavalier Marini con la sua Cara," pp. 250-253; Le notti d'amore (Trieste: Tip. Italiana, 1906); Piero Lorenzoni, Erotismo e pornografia nella letteratura italiana (Milano: il Formichiere, 1976), contiene due componimenti attribuiti al Marino: "Antonio e Cleopatra," poemetto di 14 ottave e "Indovinello del Cav. Marino," 3 quartine.

$5 \mathrm{Mi}$ rendo perfettamente conto dei limiti e dei rischi che si corrono separando nettamente lo stile del Marino dalle sue idee. Ho ben presente l'autorevole lezione del Pozzi: "Per lui in principio non sono le cose, ma le parole" (L'Adone, tomo II, pp. 767-768). Ma pure, dopo tante discussioni e ricerche sugli aspetti formali distintivi del poeta, mi è parso necessario puntare l'attenzione sull'uomo e sulle sue convinzioni. Dalla Lira all'Adone Marino si sobbarcò di tanti immani fatiche: non si investe un'intera esistenza se non si ha nulla da "dire." Lo stile del poeta poi, non è una novità, fu imitato e copiato anche da maestri valenti. La recente prova della "falsità" del "Testamento amoroso" (Angelo Colombo, Studi Secenteschi XXV, 1984, 101-22) dovrebbe far riflettere sulla presunta affidabilità totale dell'analisi stilistica. Un esame condotto nel 
territorio del "pensiero" di Marino mi è parso utile; già da queste pagine, per esempio, in cui discorro tanto dell'angoscia mariniana, la famosa "Pastorella" - lodata dal De Sanctis (Storia, cit., p. 645), attribuita al Marino anche dal Croce (Poesie varie, 1913, p. 407) e, in epoca piú recente dal Mirollo (The Poet of the Marvelous, 1963, pp. 294-301) e dal Pieri (Per Marino, 1976, pp. 58-59) sembra proprio rivelarsi un falso; la firma dell'impostore sarebbe nell'ultimo verso (luogo notoriamente previlegtiato dal poeta): "Si partí lieta, ed io restai contento". ("Poesie erotiche," in Opere del Cav. G.B. Marino, cit., pp. 513-16).

6 Abbiamo, per ora le Lettere, a cura di Marziano Guglielminetti (Torino: Einaudi, 1966) da integrare, per le corrispondenze dei contemporanei con l'Epistolario, a cura di Angelo Borzelli e Fausto Nicolini (Bari: Laterza, 1912).

7 Dal sonetto "Per la Signora N. Porta," in "Amori," Lira III, 1614. (Ediz. consultata Venezia: Ciotti, 1615, p. 55).

8 Dal sonetto "Piacere e affanno," in "Amori," Lira III, cit., p. 9.

9 In Lira III, cit., pp. 49-54.

10 In Opere scelte di Giovan Battista Marino e dei Marinisti, vol. I, "Introduzione," p. 10 .

11 E nota la formula dell'Asor Rosa: "Marino poeta servo." Egli attinge dal Getto - riportando proprio il brano citato - per appoggiare la sua tesi, in seguito vigorosamente contrastata dal Pieri in "Eros e manierismo nel Marino" (Convivium XXXVI, 1968, 453-81).

$12 \mathrm{Mi}$ riferisco alla "classica" distinzione tra "poesia" e "non poesia" applicata dal Croce al Marino in Storia dell'età barocca in Italia (Bari: Laterza, 1929).

13 "Amori notturni" è in Lira II, i.e. Rime, 1602; (ediz. consult. Rime, Venezia: Ciotti, 1602, pp. 38-42). Il "Duello amoroso" fa parte dei "Capricci" della Lira III; sebbene il primo verso sia riportato nell'indice dell'edizione del 1615, da me consultata, manca però nel volume. La leggo in "Poesie erotiche," Opere del Cav. G.B. Marino, cit., pp. 526-27.

14 L'Adone, ed. Pozzi, cit. VIII, 1.

15 I personaggi femminili sono tutti dell'Adone, il vecchio è nel madrigale XXXIV "Scherzo sopra il canto d'un vecchio sdentato," Lira II, i.e. Rime II, 1602; (ediz. colnsult. Rime, Venezia: Ciotti, 1602, p. 46).

16 Ardengo Soffici, in La voce, 31 maggio 1916. Cito dalla riproduzione dell'articolo "Principî di una estetica futurista," nel volume La cultura italiana del '900 attraverso le riviste, di G. Scalia (Torino: Einaudi, 1961), p. 589.

17 Dal sonetto "Peregrino pensier, ch'ardito e solo," in "Amorose," Lira I, ed. cit., p. 29.

18 La Sampogna, 1620; (ediz. consult. Venezia: Giunti, 1621, p. 213).

19 Marzio Pieri, "Eros e manierismo nel Marino," art. cit., p. 462.

20 Per il concetto di post-modernismo mi riferisco innanzitutto al rivoluzionario saggio di Chaïm Perelman e Lucie Olbrecht-Tyteca La nouvelle rhetorique. Traité de l'argumentation (Paris: PUF, 1958) e poi alla piú bella prova italiana di attualità del nuovo pensiero: Renato Barilli, Poetica e retorica (Milano: Mursia, 1969 e 1984).

21 Carlo Calcaterra, Il Parnaso in rivolta (Bologna: il Mulino, 19612), p. 122.

22 L'Adone, cit. VIII, 125.

$23 \mathrm{E}^{\prime}$ il secondo "idillio favoloso" della Sampogna, cit.

24 L'Adone, cit. XVI, 57.

25 Mi riferisco rispettivamente alle "lettere familiari" n. 154 (a Fortuniano Sanvitale, 1620) e n. 26 (a Bernardo Castello, 1603); in Lettere, a cura di Marziano Guglielminetti, cit.

$26 \mathrm{Si}$ può leggere in Poesie varie, a cura di Benedetto Croce, cit., pp. 353-57.

$27 \mathrm{Ho}$ già espresso diffusamente in altra sede le ragioni di questa divisione dell'Adone. Mi permetto di rinviare al mio "G.B. Marino: dal madrigale al poema grande" in Critica letteraria XIII (1985), fasc. 2, n. 47.

28 L'Adone, cit., V., 42-44. 
$29 "$. . nel profondo . . . fu indifferente a un approfondimento spirituale della Controriforma, fu schivo dall'uniformare il pensiero e i costumi alla credenza cristiana". Dal Parnaso in rivolta, cit., p. 104.

30 Il lavoro mariniano è stato riesumato da Marziano Guglielminetti. Si può leggere nel suo Tecnica e invenzione nell'opera di Giambattista Marino (MessinaFirenze: D’Anna, 1964), pp. 212-16.

31 L'Adone, cit., I, 2. 